\title{
FLEXIBLE SLIVER MODULES
}

E.A. Thomsen, J. Muric-Nesic, S. Rahman, Y. Osorio Mayon, T. Ratcliff, P. Scott, I. Skryabin, V. Everett, A. Blakers

Centre for Sustainable Energy Systems, Australian National University, Canberra, Australia

Corresponding author: ph +61 2 6125 7208, fax +61 26125 3917, email Elizabeth.thomsen@anu.edu.au

ABSTRACT: This paper presents the design, fabrication, and testing of lightweight, flexible photovoltaic modules. The modules are constructed using mono-crystalline silicon Sliver cells. The modules are designed to have a power output of at least $130 \mathrm{~W} / \mathrm{m}^{2}$; to have a very high power to weight ratio of over $150 \mathrm{~W} / \mathrm{kg}$; to be roll-able around a radius of curvature of $5 \mathrm{~cm}$ or smaller; and to be able to operate under a wide range of operating conditions, including under partial shading and after structural damage due to punctures.

Keywords: Silicon solar cell, Module, Flexible Substrate

\section{INTRODUCTION}

In recent years there has been enormous growth in the use of portable electronic devices such as mobile phones, MP3 players, laptops, cameras, and GPS units. This growth is contributing to the emerging market of flexible photovoltaics.

There are a number of technologies (including organic, dye sensitised, amorphous silicon, CdTe, and CIGS) which can potentially be used to produce flexible solar cells, and hence flexible modules. However, none of these technologies can currently match the high, stable efficiencies of crystalline silicon.

Crystalline silicon is generally unsuitable for use in flexible photovoltaic modules because it is rigid at the thicknesses currently used (several hundred microns). However, if the thickness of the silicon is reduced to below $100 \mu \mathrm{m}$, crystalline silicon becomes flexible.

Sliver cells are an example of a crystalline silicon solar cell that is naturally flexible. Sliver cells were developed at the ANU are being commercialized by Transform Solar. Rather than fabricating one solar cell from a single silicon wafer, in Sliver cell fabrication many hundreds to thousands of cells are produced from one silicon wafer, as is shown in Figure 1. Completed solar cells have dimensions of length 5-12 cm, width 0.5$3 \mathrm{~mm}$, and thickness $20-100 \mu \mathrm{m}$.

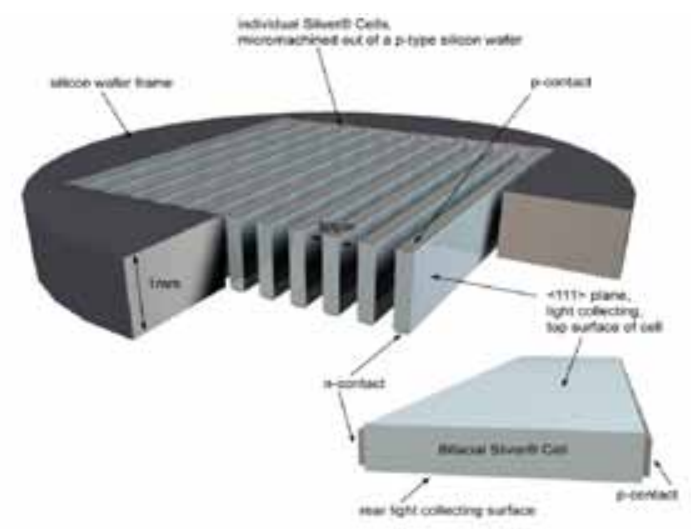

Figure 1: Structure of a wafer full of Sliver cells.

By processing several thousand Sliver cells in one wafer a much larger solar cell area is produced per unit volume of silicon than if the cell was processed using a standard fabrication, with consequent proportional reduction in processing cost and wafer starts.
In addition to their flexibility, Sliver cells have several advantageous properties for flexible photovoltaic modules. Because they are fabricated from monocrystalline silicon they have high, stable efficiencies. The elongate form factor of the cells means that when connected in series, system voltage can be rapidly built, at a rate of 5 to $10 \mathrm{~V} / \mathrm{cm}$. Hence, battery voltage can be generated in a small area, allowing cells to be incorporated into small portable electronic devices. The cells are also lightweight, allowing high power to weight ratios. Because of the fabrication method the cells are perfectly bifacial. This property allows production of fully bifacial modules capable of absorbing light from both sides.

\section{MODULE DESIGN AND FABRICATION}

A flexible photovoltaic module is likely to have different requirements to that of a standard flat plate module. These requirements may include considerations such as whether the module is roll-able, foldable, or able to conform to a surface; what current and voltage are required; a particular specified power to weight ratio; and durability under extreme conditions. In this project the target requirements included a power output of at least $130 \mathrm{~W} / \mathrm{m}^{2}, 150 \mathrm{~W} / \mathrm{kg}$, a radius of curvature of $5 \mathrm{~cm}$ or smaller, and operating temperatures between $-40^{\circ} \mathrm{C}$ and $+65^{\circ} \mathrm{C}$. However, the module design is sufficiently robust to be easily adaptable to a range of different criteria.

\subsection{Module Design}

Because of the small size of Sliver cells the electrical circuit design of these flexible modules can be substantially different to a standard module based on conventional silicon solar cells. Sliver cells can be connected in sub-module strings, and these strings can have comparable in size to a typical silicon solar cell but with substantially higher voltage and correspondingly lower current. These sub-module strings can then be connected either in series (to further build voltage), in parallel (building current and also introducing redundancy), or in any combination of series and parallel. By placing strings in parallel the module output is not limited by the cell receiving the lowest illumination.

The design and small size of Sliver cells enables them to have non-destructive reverse breakdown characteristics and also for the cells to be arranged in series-parallel circuits, allowing modules to be built without requiring bypass diodes. This property significantly simplifies the module design complexity. 


\subsection{Module Fabrication}

Flexible Sliver modules consist of Sliver cells, electrical connections, and encapsulation. All of these components need to be flexible and able to withstand long term outdoor use.

The first step in module fabrication is the creation of series strings of Sliver cells. The cells are arranged in arrays, with the arrays held together using thin, transparent tape which is able to withstand a wide range of temperatures. The tape also forms the structure for deposition of electrical interconnections.

The electrical interconnections are formed by syringe-dispensing a silver-loaded, flexible, electrically conductive paste. Conductive paste is used in preference to solder due to its higher flexibility. Sliver cells are edge-contact, with only one $\mathrm{n}$ and one $\mathrm{p}$ electrode per cell. This metallisation arrangement allows series connections to be formed simply by drawing a line of conductive paste between the right edge of one cell, and the left edge of the adjacent cell. To increase the robustness of the circuit at least two connections are made between adjacent cells.

Series strings are connected in parallel using tinned copper bus bars which are then connected to the external connections for the module. The bus bars have comparable thickness, width, and flexibility to the Sliver cells, allowing them to be easily incorporated into the module. Connections between the series strings and the bus bars are made using conductive paste. All of the elements of the circuit are shown in Figure 2.

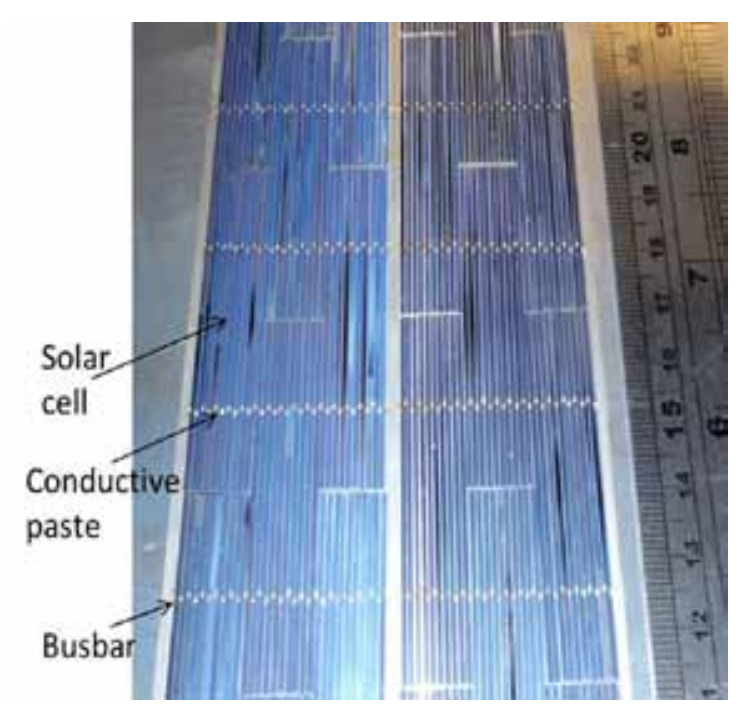

Figure 2: Image showing detail of the electrical connections between cells and banks of cells.

Once all the electrical connections are made the module is encapsulated using fluoropolymer cover sheets and optically clear two-part silicone. The final modules are around $400 \mu \mathrm{m}$ thick, and weigh close to $80 \mathrm{~g}$. Since the Sliver cells are perfectly bifacial, bifacial modules can also be made if both cover sheets are transparent. Figure 3 shows an example of a standard unifacial module, and Figure 4 shows a bifacial module.

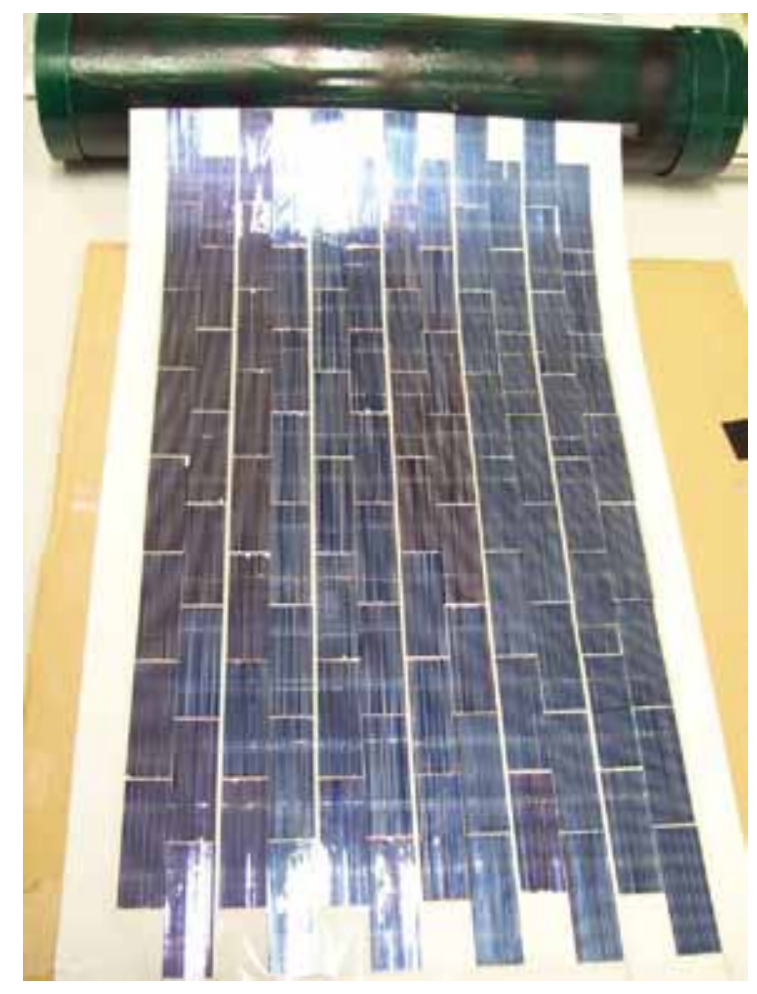

Figure 3: Image of a $0.1 \mathrm{~m}^{2}$ unifacial module.

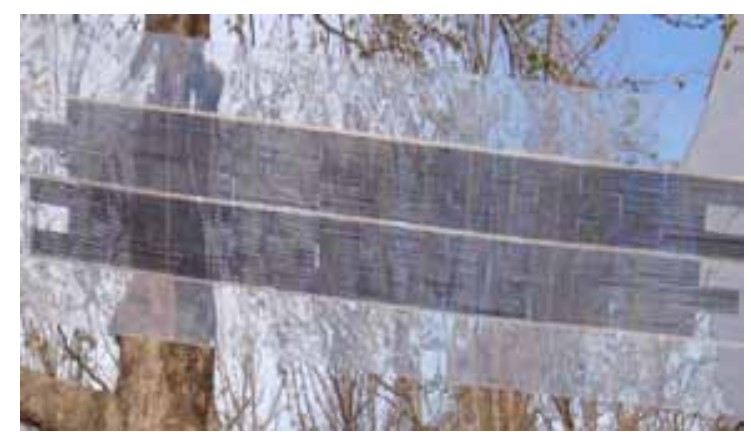

Figure 4: Image of a bifacial module, which allows absorption from both sides of the module.

\section{MODULE CHARACTERISATION}

The electrical performance of the modules was measured either outdoors, or using a solar simulator. Figure 5 shows the current-voltage curve of a representative $0.1 \mathrm{~m}^{2}$ module tested outdoors under 1.1 suns. This module had a short circuit current of $1.42 \mathrm{~A}$, open circuit voltage of $15.2 \mathrm{~V}$, fill factor of $67 \%$, and power output of $14.5 \mathrm{~W}$. The power to weight ratio of this module is $178 \mathrm{~W} / \mathrm{kg}$.

Following initial electrical testing, the modules undergo a variety of testing, including flexibility, shading, and puncture tests. The methods and results used for each of these test procedures will be described in the following sections. 


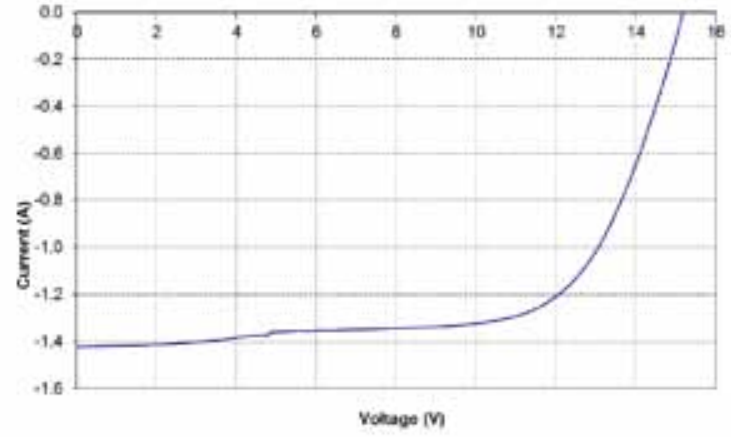

Figure 5: Current-voltage characteristics of a typical $0.1 \mathrm{~m}^{2}$ module.

\subsection{Flexibility}

There is no published standard method for testing flexible photovoltaic modules. One possible method is to mount the module on a curved surface and test the electrical performance of the module before and after flexing. An alternative method is to repeatedly flex the module to a given radius.

Figure 6 shows a purpose-built jig designed to perform repeated flexes of a module. The PVC pipe can be changed depending on the radius of curvature required. A stepper motor is used to rotate the PVC pipe, and hence flex the module. The number of flexes can be easily adjusted, with 1000 flexes generally used.

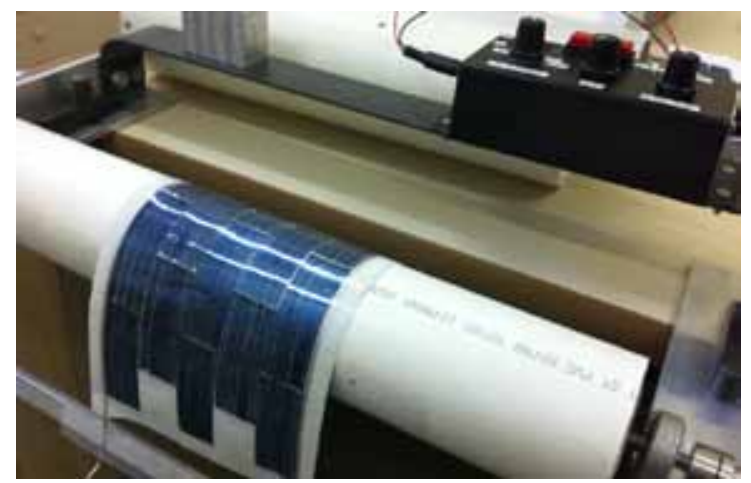

Figure 6: Image of the custom-designed flex jig.

After each set of 1000 flexes the module electrical performance wais measured and the radius of curvature of flexing was decreased by $1 \mathrm{~cm}$. The module then underwent 1000 further flexes at the smaller radius of curvature and the process was repeated down to a $2 \mathrm{~cm}$ radius of curvature. Figure 7 shows the results for the relative performance of the modules after each set of 1000 flexes.

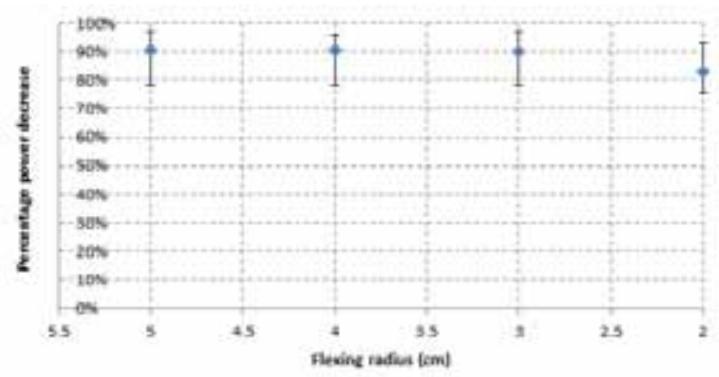

Figure 7: Relative performance of modules after flexing.1000 times.
The data in Figure 7 represents the average performance of 6 modules. For 5, 4, and $3 \mathrm{~cm}$ radii of curvature the average decrease in performance is less than $10 \%$.

\subsection{Shading}

These modules are designed to be lightweight and roll-able, allowing them to be used in a variety of environments. Hence, the modules need to be able to withstand a variety of light conditions including partial shading.

The modules were tested under a number of partial shading conditions, as shown in Figure 8, and their electrical output for each shading condition was measured.
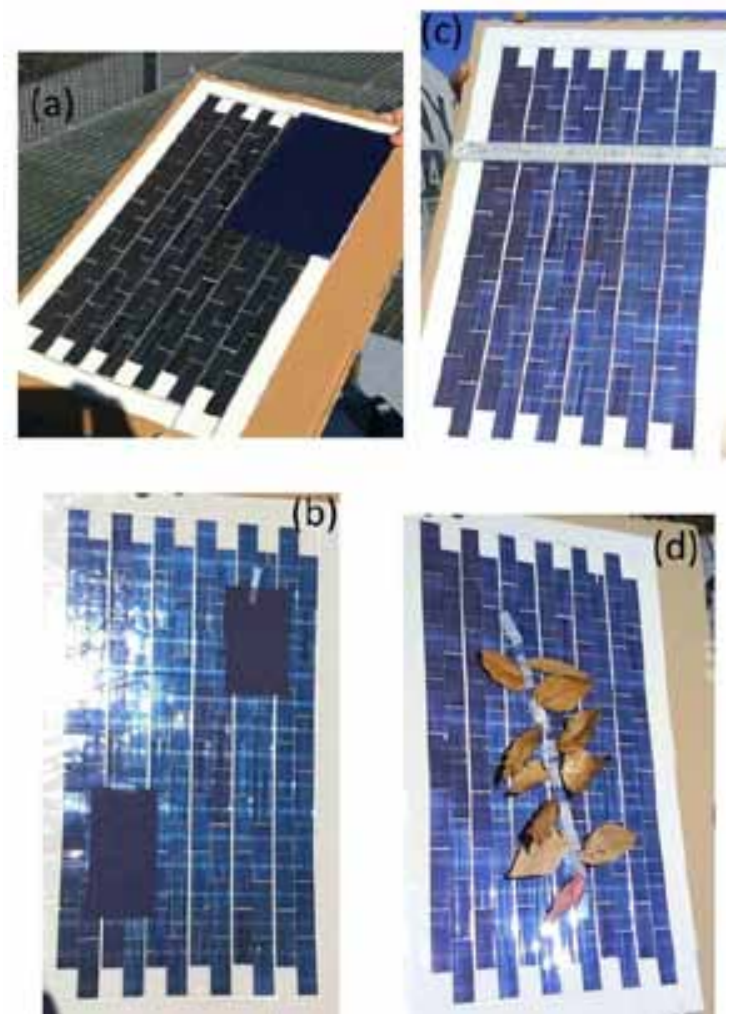

Figure 8: Images of the range of partial shading configurations used for this test.

The electrical output for each shading configuration is shown in Figure 9. In each case the shading affected both the current and the voltage. The decrease in current seen in each case was consistent with a proportional decrease in the amount of light available to the module.

The decrease in open circuit voltage is likely to be primarily due to fewer illuminated cells in certain series strings. In general the fill factor of the modules was not significantly affected by the shading. However, in some cases the shape of the curve did change slightly; presumably this was due to current mismatch. In each case the efficiency, corrected for the reduced active area, was within one percentage point of the unshaded performance. This demonstrates the robustness of the series-parallel circuit topology. 


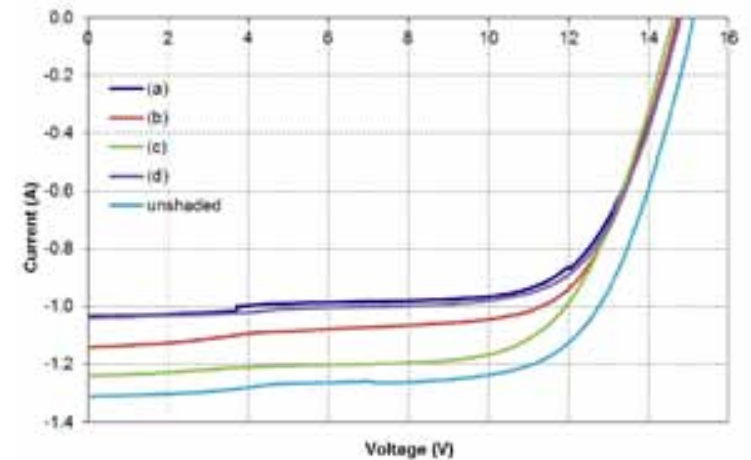

Figure 9: Current-voltage characteristics of shaded (a to d corresponding to configurations illustrated in Figure 8) and unshaded module.

The tolerance of the modules to partial shading is due to the small size of the cells, the elongate form factor, and the series-parallel circuit configuration. All these features allow the rapid building of voltage within a small area. By building up the required voltage in a small area, series-parallel circuit designs can be used to maximum effect. These designs allow shading of one area of the module without limiting current through the whole module, with the modules continuing to operate at close to optimum conditions, albeit with a reduced area available for current generation, when shaded.

\subsection{Puncturing}

The series-parallel circuit design also allows the modules to be resistant to puncturing. Punctures can occur in several regions of the module: on cells in one series string, on cell interconnections, on bus bars, or on some combination of these regions. Punctures to cells are the least destructive of these types, while puncture to the bus bars are the most destructive.

For a module of area approximately $80 \mathrm{~cm}^{2}$, each puncture of diameter $7 \mathrm{~mm}$ in a cell-only area decreases the total power output by between $1 \%$ and $3 \%$. This decrease is partly due to the decreased effective area of the module, partly due to increased recombination at the broken surfaces, and partly due to consequent current mismatch.

Puncturing the cell interconnections not only results in a loss of area, increased recombination, and current mismatch; but also reduces the number of pathways for the current to flow. Figure 10 shows a module with punctures made to 3 of the series interconnection strips, 2 of which are on the same series string. This module suffered a total decrease in power of $30 \%$, primarily due to loss in current.

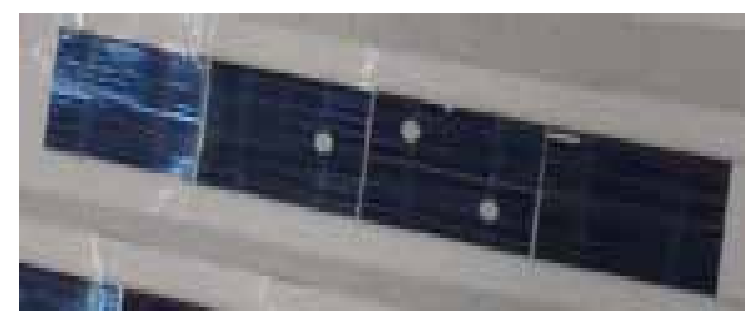

Figure 10: Image of a module with puncture damege on cell interconnections.
Puncturing a bus bar effectively removes part of the module from contributing to the power output. Depending on the placement of the puncture anything from one series string to the whole module can be removed. However, in a $0.1 \mathrm{~m}^{2}$ module the area covered by bus bars is approximately $3 \%$ of the total surface area, making the likelihood of puncturing a bus bar very low.

The most likely area for a puncture to occur is on a cell or group of cells, and this has minimal effect on the overall output.

\section{CONCLUSION}

Flexible photovoltaic modules based on monocrystalline silicon Sliver cells have been designed, fabricated, and tested. The modules have an output of over $13 \mathrm{~W}$ for a $0.1 \mathrm{~m}^{2}$ module; have high power to weight ratios of over $150 \mathrm{~W} / \mathrm{kg}$; and are robust to a variety of environmental conditions, including partial shading and puncturing.

\section{ACKNOWLEDGEMENTS}

The CTD Program, managed by the Defence Science and Technology Organisation, assists in the improvement of priority Defence capability by providing Australian industry with an opportunity to demonstrate their technology. This enables Defence to assess the potential of the technology to enhance military capability as well as the likely risks associated with acquiring the technology. This work was funded by the Department of Defense under the DSTO CTD program. 